\title{
Estudo Prospectivo sobre Sistema de Controle e Aceleração em Elevadores
}

\author{
Prospecting Study About Control System and Acceleration in Elevators
}

\author{
Gisele Cristina Borges ${ }^{1}$ \\ Ana Carolina Nerva Blumm ${ }^{1}$ \\ Eduardo Henrique da Silva Figueiredo Matos ${ }^{1}$ \\ Carolyne Caetano Gonçalves ${ }^{1}$ \\ Wina Eleana Lages Pereira ${ }^{1}$ \\ Roosevelt Tomé Silva Filho ${ }^{1}$ \\ Paulo Gustavo Barboni Dantas Nascimento ${ }^{1}$ \\ Adriana Regina Martin ${ }^{1}$ \\ ${ }^{1}$ Universidade de Brasília
}

\begin{abstract}
Resumo
O presente estudo pretende mostrar, por meio da prospecção tecnológica, uma análise comparativa e analítica de tecnologias que utilizam arquiteturas reconfiguráveis com sistemas de controle e aceleração para o controle do tráfego de usuários em elevadores comerciais, visando a reduzir o tempo de espera de viagem dos usuários e diminuir o consumo de energia. Para esse levantamento, foram realizadas pesquisas na base de patentes Patentscope WIPO, consultas na base de artigos Web of Science, bem como uma análise de mercado e avaliação da maturidade tecnológica desse sistema de controle. A patente registrada no INPI pela Universidade de Brasília sob o $n^{\circ}$ PI 9500121-1 foi usada como objeto de estudo. Foram encontradas 662 patentes e 113 artigos através de buscas por palavras-chave, sugerindo que essa tecnologia está madura e, portanto, bem estabelecida no mercado. Assim, foram sugeridas novas aplicações para a tecnologia de forma a justificar a manutenção da patente, além de uma análise FOFA demonstrando os pontos fracos e fortes da tecnologia.
\end{abstract}

Palavras-chave: Controle. Aceleração. Elevador.

\begin{abstract}
The present study shows by technology prospecting a comparative and analytical analysis of technologies that use reconfigurable architectures with control system and acceleration in order to control passengers traffic in commercial elevators, seeking to reduce users waiting time and also decrease energy consumption. For this study, research in patent database Patentscope WIPO, consults at Web of Science for scientific articles, besides a market analysis and evaluation of technology maturity were made. The patent registered by University of Brasilia, $n^{\circ}$ PI 9500121-1 was used as an object of study. There were found 662 results for patents and 113 for articles in the search for keywords, suggesting that this technology is mature, therefore new applications for this technology were suggested in order to justify the maintenance of this patent, besides a SWOT analysis proving its weak and strong points.
\end{abstract}

Keywords: Control. Acceleration. Elevator.

Área tecnológica: Indústria de Elevadores. 


\section{Introdução}

O mundo possui mais de 12 milhões de elevadores que carregam cerca de 1 bilhão de pessoas por dia (CABRAL, 2016). Com tantas pessoas utilizando elevadores, é importante analisar as instalações sob o ponto de vista econômico, no que tange à custos de energia, operação $e$ manutenção, bem como a satisfação dos usuários em relação ao conforto e tempo de espera. Sendo assim, a invenção aqui estudada, "Sistema de controle distribuído de grupo de elevadores usando dispositivos reconfiguráveis", propõe um hardware capaz de otimizar a utilização de tais meios de transporte.

A invenção propõe uma configuração de computador pessoal com hardware integrado a sistema de controle de grupo de elevadores, rede de conexão e controle local. Tal configuração permite a gestão do conjunto desses equipamentos de um edifício e a aceleração do processo de transporte vertical. O primeiro controle faz análise de tráfego existente, a partir de dados recebidos dos elevadores. Em seguida, define o algoritmo que o elevador utilizará. Então, o controle local executa o algoritmo, determinando em qual andar deve parar, quando seus motores devem funcionar e o momento de abertura de portas (UNIVERSIDADE DE BRASÍLIA, 2010).

A patente foi registrada pelo Instituto Nacional da Propriedade Industrial (INPI) sob o código PI0803135-5, cujo depósito ocorreu em 2008 e publicação em 2010, e ainda não foi concedida. Seu registro é renovado anualmente pelo Centro de Apoio ao Desenvolvimento Tecnológico (CDT) da Universidade de Brasília. Contudo, após 10 anos da data de depósito e do advento de novas tecnologias relacionadas a sistema de controle e aceleração de elevadores, questiona-se a necessidade de tal patente, haja vista que muitas dessas invenções tornarem-se obsoletas e sem aplicabilidade.

Na nova realidade imposta pela Emenda Constitucional (EC) n. 95, de 2016 (BRASIL, 2016), limitando os gastos públicos, uma nova era de eficiência e planejamento de gastos deve ser estabelecida. Neste sentido, a qualificação do pedido de proteção industrial é justificada na perspectiva do gerenciamento dos ativos de propriedade industrial de um Núcleo de Inovação Tecnológica. O Gráfico 1 apresenta uma evolução temporal dos gastos do CDT junto ao INPI. No novo contexto apresentado pela EC 95, esta evolução deve ser revista e práticas efetivas e eficientes quanto à gestão de ativos de propriedade industrial devem ser adotadas.

A prospecção tecnológica é uma ferramenta que deve auxiliar essa gestão. Dessa forma, este estudo tem como objetivo verificar o surgimento de novas tecnologias relacionadas a sistemas de controle em elevadores com dispositivos reconfiguráveis, por meio de buscas em bancos de dados de patentes e artigos, na procura de patentes e artigos depositados até os dias atuais. Além disso, pretende analisar as condições de mercado e o grau de maturidade para essa tecnologia, a fim de justificar os recursos financeiros destinados à manutenção deste pedido de patente até sua eventual concessão. 
Gráfico 1 - Gastos diretos do CDT da Universidade de Brasília, efetuados ao INPI como: Outros Serviços de Terceiros - Pessoa Jurídica

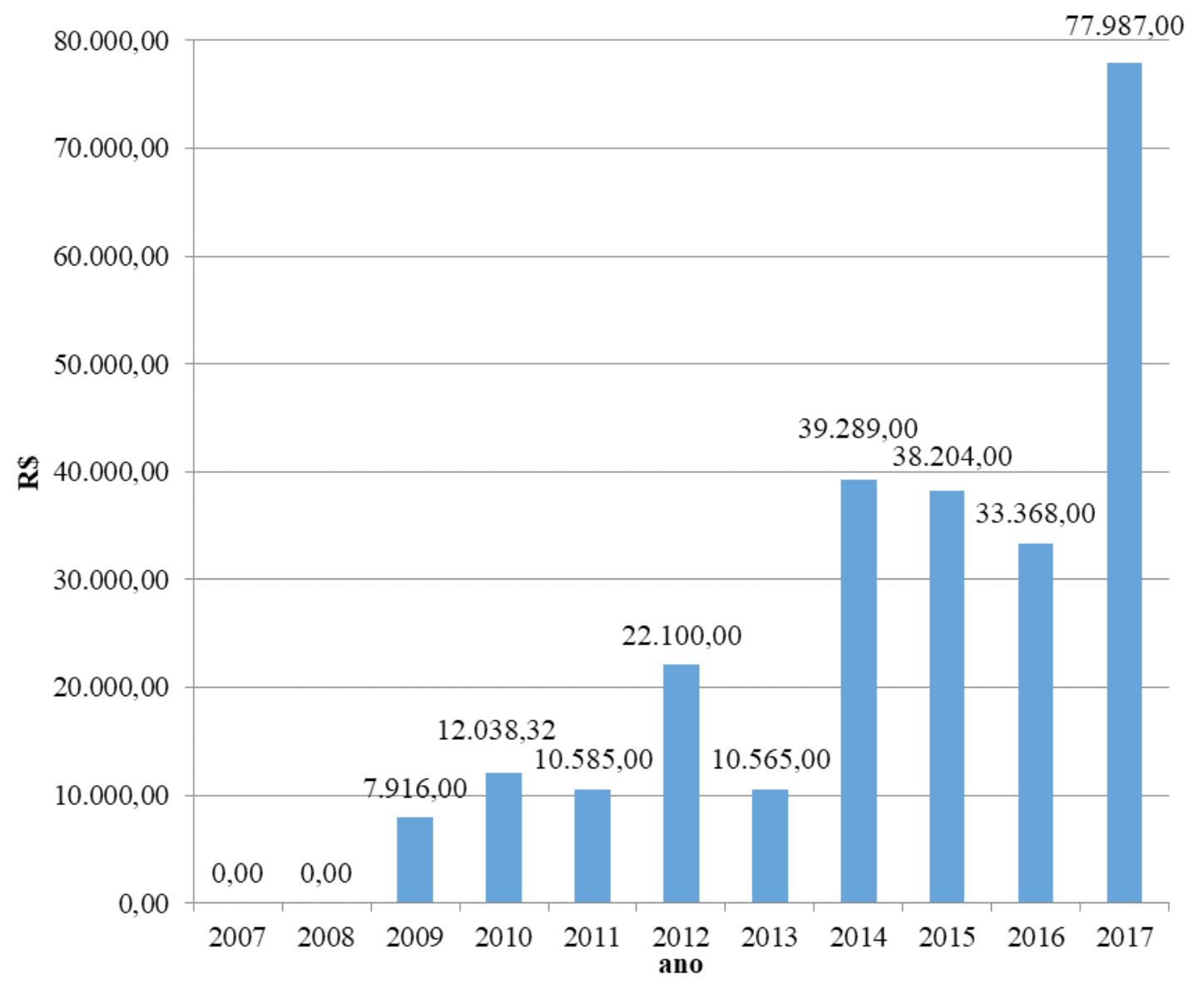

Fonte: Elaborado pelos autores deste artigo (2018) com dados retirados do Portal de Transparência (Brasil, 2009)

\section{Metodologia}

O presente estudo foi realizado por meio de pesquisa exploratória para entender a patente, verificar o grau de inovação e os investimentos realizados na tecnologia citada. Também foram realizadas pesquisas em bases de patentes e artigos para a análise da prospecção tecnológica e definição da maturidade tecnológica.

Para as buscas, foram definidas palavras-chaves que resumiam o escopo da tecnologia em nomes comuns, isolados e/ou agrupados com diferentes combinações e conectores. A escolha das palavras-chave foi baseada nas principais palavras presentes no texto da patente da tecnologia em questão, as quais representam a aplicabilidade e principais objetivos dessa tecnologia. Foram utilizadas as plataformas Patentscope WIPO e Web of Science, mecanismos de pesquisa gratuitos, sob os quais foram aplicadas combinações de palavras chaves. A pesquisa foi realizada nos meses de maio e junho de 2018. 
Com o objetivo de obter uma busca mais refinada, foi realizada uma pesquisa avançada utilizando um filtro pelas classificações B66B 1/24 e B66B 1/28 e uma combinação de palavras-chave. Essas classificações correspondem a sistemas de controle e aceleração, sendo segmentada por "B", que são Operações de Processamento, Transporte; "66" Içamento, levantamento, rebocamento; $e$ "B" Elevadores, Escadas rolantes; compondo a combinação B66B.

Na base de dados do Patentscope (WIPO) pelo campo "Title" ou "Title Abstract" foi feita a busca pelas combinações demonstradas na Tabela 1, com data de publicação de 01.01.2008 a 31.12.2017.

Utilizando as palavras-chave "(Elevator OR lift) AND controll*" foram encontrados 35.743 resultados de patentes. Refinando a busca, reduziu-se a chave de busca para "Elevator AND controll*", na qual foram encontradas 12.376 patentes relacionadas. Por fim, realizou-se busca avançada com as palavras-chave "(elevator OR lift) and controll* and accel*)", na qual foram encontrados 662 resultados de patentes. As palavras chave "controll*" e "accel*", uma vez que contêm o asterisco (*), permitem que palavras derivadas destas sejam encontradas na busca, por exemplo, "controlling", "controlled", "acceleration" e "accelerated", além de outras.

Tabela 1 - Estratégia de busca de patentes e números de resultados por busca

\begin{tabular}{cc} 
Combinação & Resultado \\
(elevator OR lift) AND controll* & 35.743 \\
elevator AND controll** & 12.376 \\
(elevator OR lift) and controll* and accel*) & 662 \\
\hline
\end{tabular}

Fonte: Elaborado pelos autores deste artigo (2018)

A partir dos resultados, foi possível analisar a invenção de acordo com os critérios de inovação e modernização das tecnologias utilizadas para controle e aceleração do transporte em elevadores para aferir a evolução tecnológica. Os resultados tornaram possível a análise da maturidade tecnológica da invenção. Então, foi realizada uma análise de mercado, que foi consolidada numa matriz FOFA.

\section{Resultados e Discussão}

Com os resultados encontrados por meio de uma busca na base de dados Patentscope WIPO foi possível realizar uma pesquisa pelas palavras-chave "(elevator OR lift) and controll* and accel*)", cujos resultados são apresentados no Gráfico 2. É importante ressaltar que, apesar da redução de registros em 2011 e 2012, a linha de tendência demonstra um potencial de crescimento para os próximos anos. 
Gráfico 2 - Resultados do número de patentes depositadas entre 2008 e 2017

\section{Patentes registrados por ano}

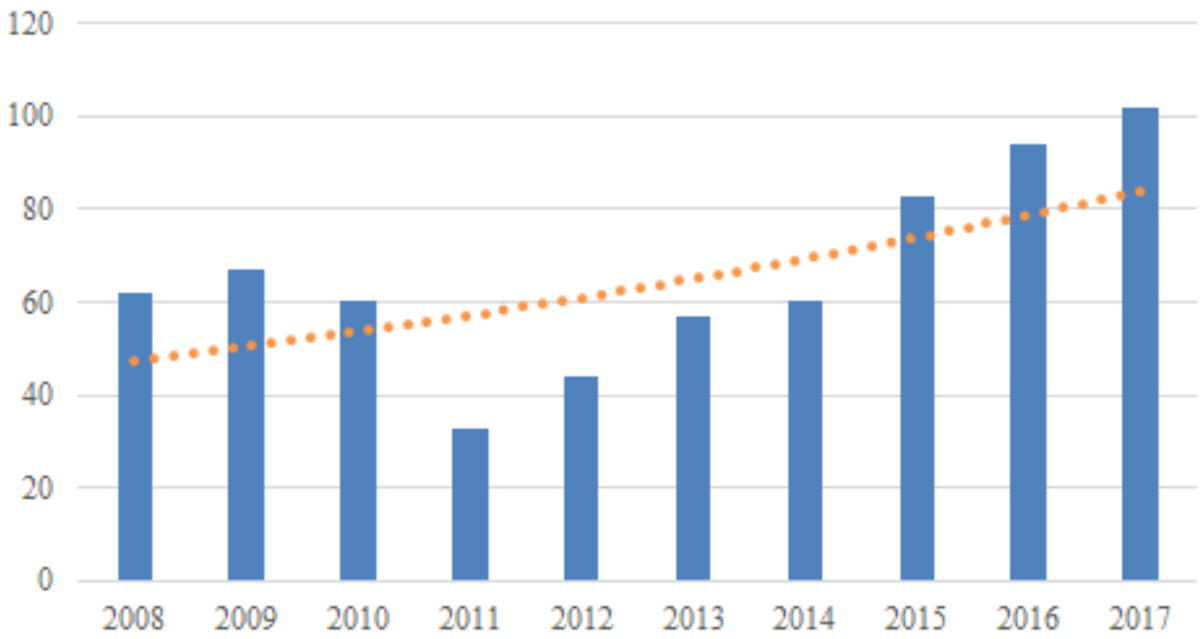

Fonte: Elaborado pelos autores deste artigo (2018)

Em relação ao número de países depositantes foram encontrados 662 resultados, tendo como principais países depositantes a China, Japão e Estados Unidos, países em que também se encontra a maior parte das grandes empresas relacionadas à fabricação de elevadores, bem como de sistema de controle e aceleração para esse meio de transporte vertical.

Gráfico 3 - Resultados do número de países depositantes de patentes e a quantidade de patentes depositadas

\section{Países}

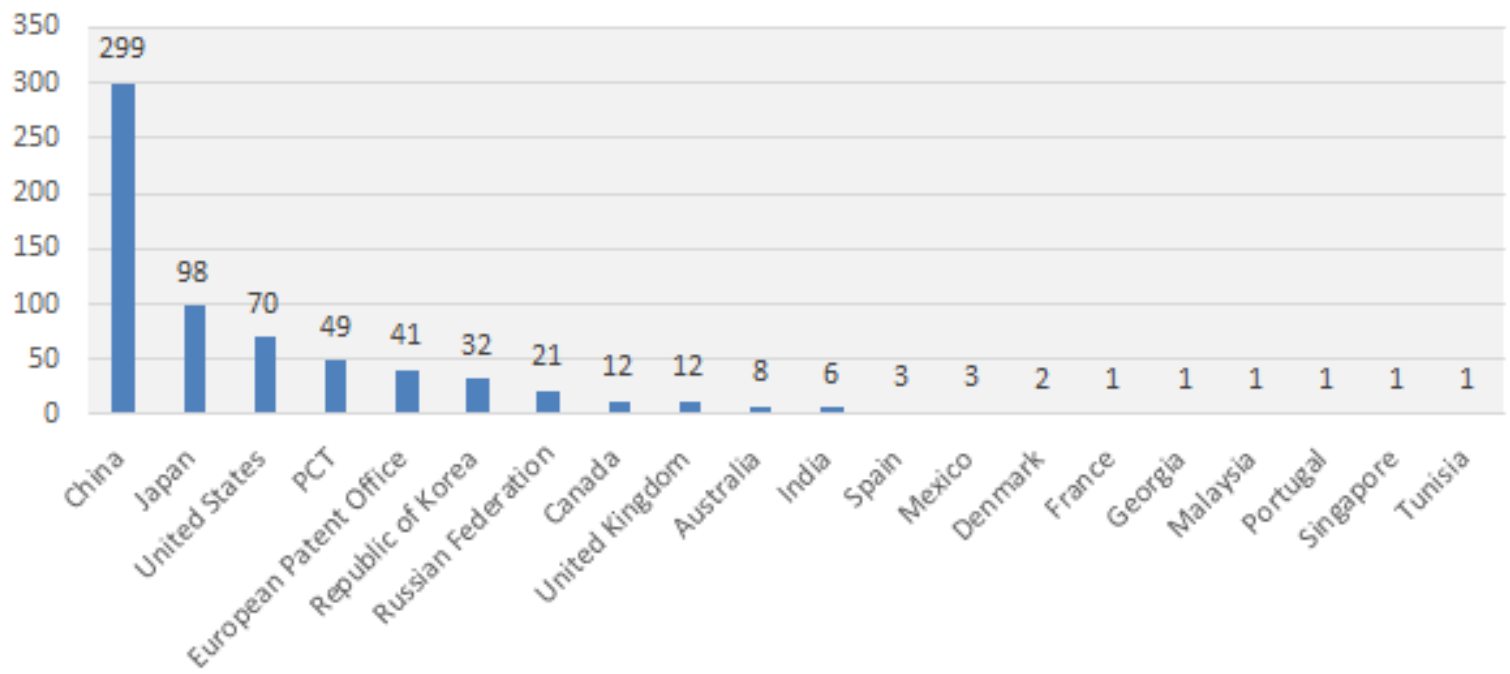

Fonte: Elaborado pelos autores deste artigo (2018)

A busca realizada por classificação (IPC) retornou várias classificações para essa tecnologia. No entanto, os dois segmentos que apresentaram mais evidência foram o B66B, que retornou 190 registros e o F02D, resultando em 37 registros, o que sugere que a tecnologia de controle de aceleração também pode ser utilizada na engenharia mecânica afunilando para o segmento de motores para combustão em geral, como é o caso da empresa israelense Aquarius Engines, 
Ltda., que inovou no mercado automobilístico no ano de 2016 ao produzir motores com capacidade de reduzir com eficácia o consumo de combustível.

Gráfico 4 - Resultados do número de patentes depositadas por IPC

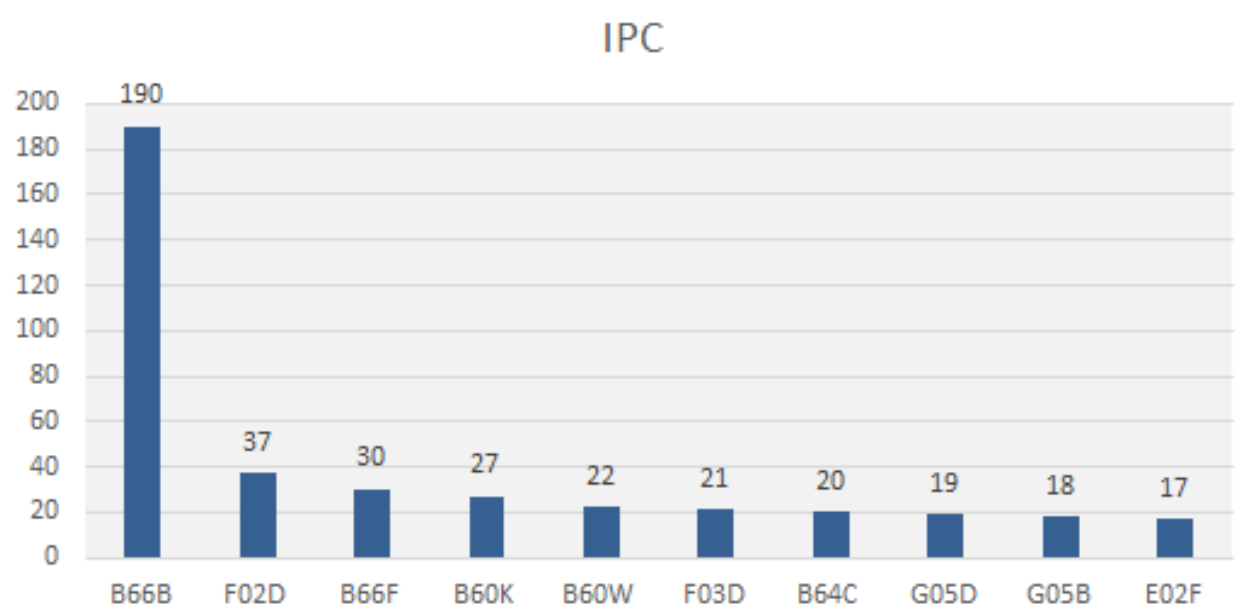

Fonte: Elaborado pelos autores deste artigo (2018)

Foi realizada uma consulta, em maio de 2018, com o inventor da patente PI0803135-5, o Professor Doutor Maurício Ayala Rincon, Titular em Ciência da Computação da Universidade de Brasília, a fim de melhorar o entendimento da invenção. Segundo o inventor, ao longo de dez anos houve uma modernização no uso de tecnologias por hardware, que possibilita produzir ou alterar facilmente sua configuração, e o diferencial é que é uma computação reconfigurável e mais segura. Essas tecnologias são chamadas de FPGA (Field-programmable gate array), que têm sido aplicadas em transportes de elevadores verticais e horizontais, saúde e robótica.

Vale ressaltar que essa tecnologia é melhor aplicada em prédios comerciais com intenso tráfego no transporte vertical, pois permite reavaliar o gerenciamento de tráfego diário ou anual para reprogramar a rotina de tráfego de acordo com o histórico do prédio, isto é, definir o melhor horário de pico de entrada e saída de usuários nas diferentes horas do dia, otimizar a viagem para andares mais acessados, visando a eficiência no atendimento e economia de energia, ou atender à mudança de usuário do prédio (UNIVERSIDADE DE BRASÍLIA, 2010).

\subsection{Análise da Maturidade Tecnológica}

Em se tratando de análise de maturidade tecnológica, vale considerar o Technology Readiness Level (TRL), que é um indicador do nível de desenvolvimento de uma tecnologia. Foi desenvolvido pela NASA (National Aeronautics and Space Administration) nas décadas de 1970 e 1980, e atualmente é amplamente utilizado por indústrias como uma ferramenta de gestão de risco para a produção de um produto. É uma ferramenta muito importante para avaliar a maturidade tecnológica de um produto (NASA, 2018).

Com o objetivo de avaliar a pesquisa e desenvolvimento (P\&D) na área de sistemas de controle e aceleração do transporte em elevadores, foi realizada uma busca em um banco de dados de artigos, no sítio Web of Science, de forma a verificar se a tecnologia em questão continua sendo objeto de estudo e se vem recebendo incentivos de pesquisa. 
Na base de dados Web of Science foram pesquisadas as palavras-chave pelos campos "Title" e "Title Abstract" por "(elevator or lift) and controll" and accel" e pelo ano de publicação "Publication Date", de 01.01.2008 a 31.12.2017, resultando em 113 artigos, distribuídos conforme demonstrado no Gráfico 5.

Gráfico 5 - Artigos por país - base de dados da Web of Science

\section{Artigos}

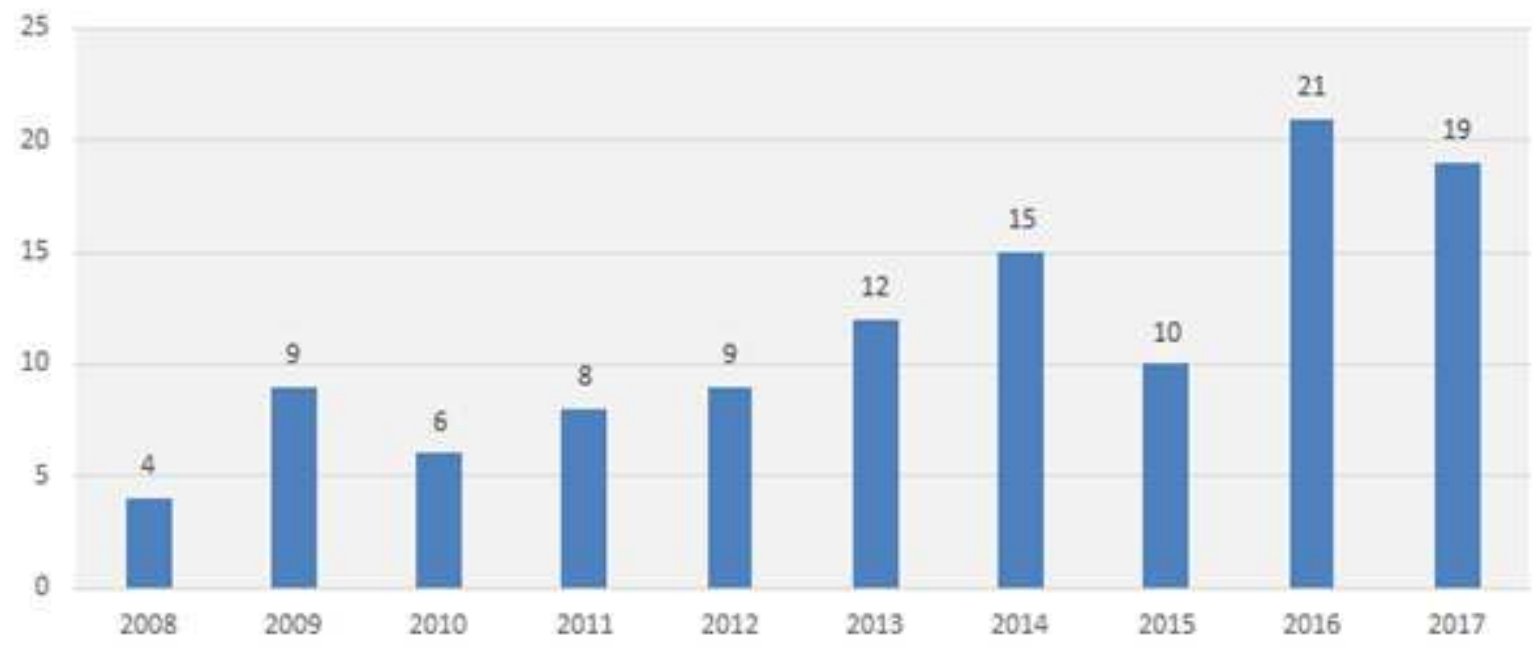

Fonte: Elaborado pelos autores deste artigo (2018)

A fim de avaliar a maturidade tecnológica da tecnologia de sistemas de controles utilizando sistemas reconfiguráveis e aceleração em elevadores, foi realizada uma comparação entre os dados encontrados na base de dados de patentes, Patentscope WIPO, e na de artigos, Web of Science. Dessa forma, é possível avaliar se a tecnologia estudada ainda está em fase de P\&D, recebendo grandes incentivos em pesquisa, se é um produto em ascensão, ou se já é uma tecnologia estabelecida, para a qual se pode encontrar muitas patentes relacionadas, em diversos países, sendo amplamente produzida pelas indústrias do ramo.

Para melhor visualização do parâmetro de maturidade tecnológica, foi elaborado um gráfico agrupado por número de patentes e artigos encontrados, respectivamente, no Patentscope WIPO e no Web of Science entre os anos de 2008 e 2017.

Por meio dos resultados é possível identificar e compreender que a tecnologia de controle e aceleração para elevadores se encontra bem estabelecida no mercado em que está inserida, portanto é uma tecnologia madura e que já vem sendo aplicada e fabricada há algum tempo pelo mercado de empresas de elevadores. É possível inferir esse dado, uma vez que foi encontrado um número maior de patentes em relação ao número de artigos, 662 e 113, respectivamente. 
Gráfico 6 - Comparação de Artigos e Patentes entre as bases de dados Patentscope e Web of Science por ano

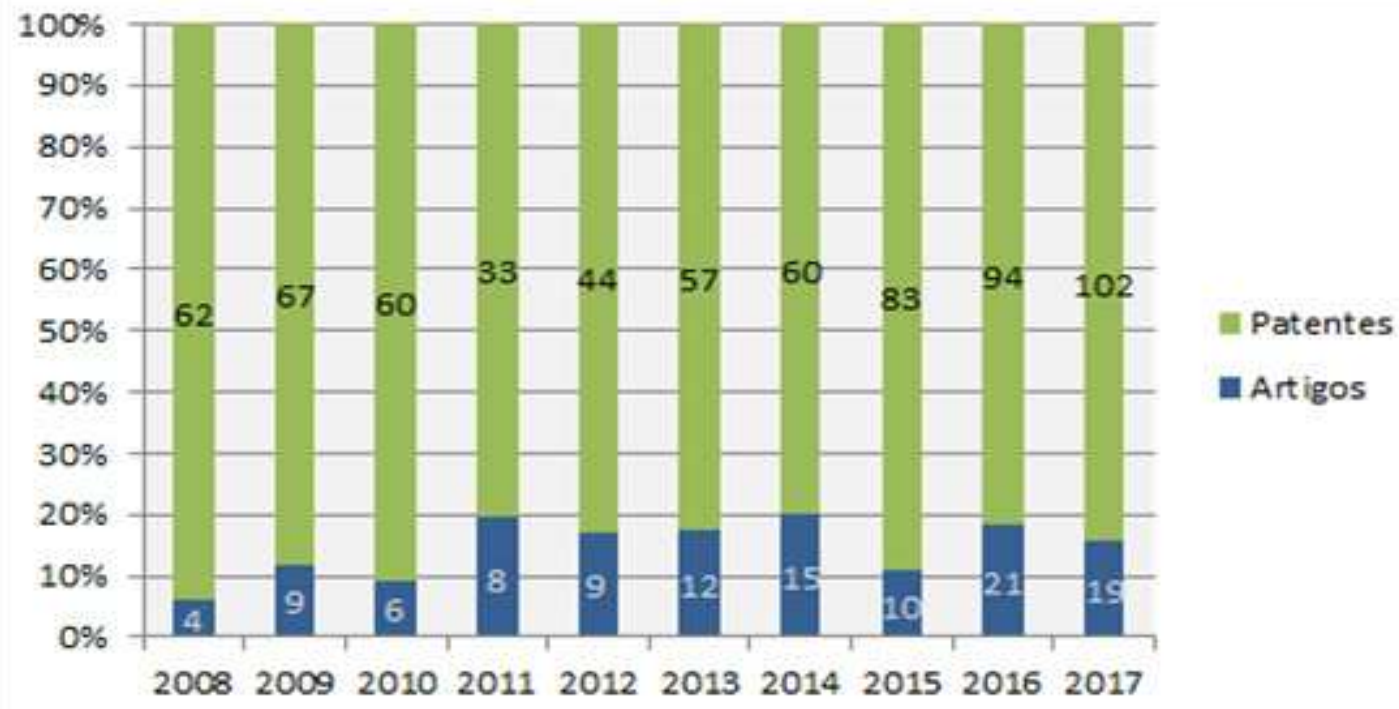

Fonte: Elaborado pelos autores deste artigo (2018)

Considerando-se que a tecnologia identificada pela patente PI0803135-5 só foi produzida a nível de protótipo, ou seja, um modelo que consiste em um simulador para testar o bom desempenho do sistema de controle de elevadores através de dispositivos reconfiguráveis, sua classificação TRL seria a TRL4, na qual é realizada a validação da tecnologia e seus componentes em ambiente laboratorial (NASA, 2018), com o objetivo de testar se todos os dispositivos funcionam corretamente em conjunto, cumprindo o propósito para que foram desenvolvidos, isto é, verificar sua usabilidade numa plataforma de reconfiguração por hardware, o que resultou numa performance consideravelmente flexível e modular.

Contudo, quando é feita a análise dessa tecnologia no contexto em que ela está inserida, juntamente com a sua análise de mercado, é possível verificar que os produtos relacionados a controle e aceleração de transporte em elevadores já estão sendo amplamente produzidos pelas fábricas de sistemas para elevadores, dessa forma consistindo em uma tecnologia bem estabelecida no mercado. Assim, considerando-se que a tecnologia para ser comercializada precisa ser testada em ambiente relevante, garantindo a sua funcionalidade e segurança para os usuários, é possível inferir que a tecnologia, de modo geral, já possui várias opções no mercado.

Analisando a pesquisa de mercado realizada, além da entrevista realizada com um dos inventores, o Professor Doutor Maurício Ayala Rincon, foi possível verificar que, atualmente, existem sistemas semelhantes ao da invenção no mercado, ao menos no que se refere à otimização do serviço, contudo a análise da aplicação da patente depende da verificação do hardware utilizado no sistema eletrônico do elevador.

As principais empresas globais de elevadores têm investido em modernização tanto tecnológica quanto estética em equipamentos e sistemas. A modernização visa a melhorar o tempo 
de espera, garantir a segurança e conforto dos usuários, além de economia no consumo de energia e redução nos custos na manutenção. Nesse conjunto de busca por palavras-chaves as empresas que se destacaram foram a Hitachi Ltda. e a Mitsubishi Electric Corporation.

A empresa Hitachi Ltda. vem investindo fortemente em novas tecnologias para elevadores e sai na frente de outras concorrentes no mercado internacional. Em junho de 2017 testou o elevador mais rápido do mundo em Guangzhou, na China, no famoso CTF Finance Centre, com 530 metros de altura. Com base em melhorias adicionais nos sistemas de controle e segurança, o elevador alcançou uma velocidade de 1.260 metros por minuto, ou seja 75,6 km por hora (HITACHI, 2017).

Outra campeã em tecnologia de ponta é a japonesa Mitsubishi Electric Corporation. Um dos projetos de grande visibilidade mundial foi a implantação de alta tecnologia no Build Elevator Training Center at Inazawa Works, no Japão, inaugurado em novembro de 2017. O investimento foi mais de 12 bilhões de dólares. O projeto teve como expectativa a segurança e o conforto dos passageiros.

\subsection{Análise FOFA}

Considerando a análise da maturidade tecnológica da invenção em destaque e a perspectiva de mercado apresentada, é possível consolidar as visões numa matriz FOFA, que representa as forças, fraquezas, oportunidades e ameaças existentes. Nesse sentido, a seguir é apresentada a FOFA para a invenção, na Matriz 1.

Observando-se a matriz, é possível identificar que os quatro fatores avaliados se encontram equilibrados, porém há uma leve preponderância dos pontos fortes. Isto se dá pela maturidade da tecnologia ser alta, enquanto o mercado ainda possui poucos concorrentes. Neste sentido, ressalta-se que durante a entrevista com o inventor foi destacada a falta de investimento para a comercialização do produto, o que justifica que, mesmo com as forças e as oportunidades existentes, a invenção não tem grande participação no mercado.

Tal observação vem de encontro ao Marco Legal da Ciência, Tecnologia e Inovação (Lei 13.246/2016), que dentre outros pontos ressalta a importância de o aspecto de comercialização ser aplicado nos desenvolvimentos em âmbito universitário e ser considerado para patenteamento. Ainda com relação ao Marco, ele ressalta a interligação da hélice tripla (universidade, governo e indústria) no sentido de que a invenção aqui destacada possui potencial de mercado, porém não chegou a indústria.

Assim sendo, considerando-se que a tecnologia está madura e possui potencial de mercado, sugere-se que a invenção seja licenciada para grandes empresas do setor de elevadores. Isso porque, uma vez que o mercado possui poucos participantes, é importante que se considere um parceiro forte para seu lançamento. Para este parceiro, ressalta-se o aspecto de que o licenciamento de tecnologias já desenvolvidas é mais vantajoso que a criação e a manutenção de setores de P\&D. Sendo assim, o licenciamento da tecnologia ressalta um contrato entre o inventor e a empresa licenciante, em uma relação ganha-ganha, ou seja, vantajosa para ambos. 
Quadro 1 - Matriz FOFA para a avaliação da tecnologia

\begin{tabular}{|c|c|}
\hline $\begin{array}{l}\text { Forças } \\
\text { Menor consumo de potência de elevadores; } \\
\text { Fácil manutenção por utilizar placas } \\
\text { reconfiguráveis que permitem configurações } \\
\text { e substituições mais fáceis; } \\
\text { Interface de monitoramento dos elevadores } \\
\text { desenvolvida em plataforma livre; } \\
\text { Possibilita o controle de grupo para } \\
\text { análise das condições de tráfego e } \\
\text { distribuição da carga computacional; } \\
\text { Fácil percepção do algoritmo pelas } \\
\text { unidades locais dos elevadores; } \\
\text { Tecnologia madura, pronta para o mercado. }\end{array}$ & $\begin{array}{c}\text { Fraquezas } \\
\text { Necessidade de aquisição de um } \\
\text { quantitativo elevado de controladoras } \\
\text { FPGA; impactando o custo da solução; } \\
\text { As controladoras utilizadas pela invenção } \\
\text { não são produzidas localmente, } \\
\text { necessitando sua importação e sujeitar-se a } \\
\text { eventual descontinuidade no fornecimento } \\
\text { de peças de reposição do dispositivo; } \\
\text { A evolução tecnológica dos dispositivos } \\
\text { FPGA utilizados implica uma reavaliação } \\
\text { do uso da tecnologia, a partir da adoção } \\
\text { de placas reconfiguráveis mais modernas. }\end{array}$ \\
\hline $\begin{array}{c}\text { Oportunidades } \\
\text { Maior eficiência no transporte } \\
\text { vertical de passageiros; } \\
\text { Mercado com poucos fornecedores; } \\
\text { Uso de plataformas livres, possibilitando o } \\
\text { desenvolvimento de sistemas escaláveis e flexíveis; } \\
\text { Solução aderente às tecnologias de } \\
\text { elevadores antigos e novos, evidenciando } \\
\text { alta capacidade de adoção pelo mercado; } \\
\text { Aderência da tecnologia patenteada } \\
\text { para elevadores de transporte vertical } \\
\text { não apenas de pessoas. }\end{array}$ & $\begin{array}{c}\text { Ameaças } \\
\text { Tecnologia já incorporada nos } \\
\text { elevadores modernos; } \\
\text { Novos fornecedores e } \\
\text { concorrentes internacionais; } \\
\text { Necessidade de certificação } \\
\text { do equipamento; } \\
\text { Setor rigidamente normatizado e } \\
\text { controlado por órgãos reguladores. }\end{array}$ \\
\hline
\end{tabular}

Fonte: Elaborado pelos autores deste artigo (2018)

\section{Considerações Finais}

A tecnologia patenteada sob o código PI 0803135-5, depositada junto ao INPI em 2008, apresenta uma inovação que privilegia a redução do consumo de energia do sistema de elevadores, acelerando o transporte e reduzindo o tempo de espera de seus usuários. Desta forma, a tecnologia visa a otimizar o transporte vertical de pessoas através de um sistema de controle de grupo distribuído para elevadores utilizando dispositivos reconfiguráveis.

Como apresentado neste texto, as inovações no setor de transporte vertical estão em constante evolução, a partir de investimentos e modernização em tecnologias semelhantes à invenção. Vale ressaltar que, além da eficiência operacional e conforto de seus usuários, a tecnologia na área vem buscando incorporar aspectos sustentáveis que reduzam o impacto ambiental.

Os resultados encontrados foram obtidos através de buscas por palavras-chave como "elevators", "controll”, "accelaration" etc., de forma que as buscas foram refinadas através de códigos IPC de maior relevância, visando a levantar o quantitativo de documentos recuperados nas diversas estratégias de busca.

Para um maior aprofundamento, foi realizada uma análise do grau de maturidade tecnológica (TRL) da invenção em foco, partindo de uma prospecção em bases de artigos e patentes nacionais e internacionais, e também realizada uma entrevista com um dos inventores da patente, Professor Doutor Maurício Ayala Rincon, com o objetivo de otimizar o entendimento. Tal prospecção auxiliou na compreensão da tecnologia em seu contexto e direcionou as buscas em relação aos principais fabricantes e países mais desenvolvidos em tecnologias semelhantes. 
Dessa forma, este artigo buscou entender a invenção identificada pela patente PI 0803135 5 , contextualizando a sua relevância para o momento em que foi desenvolvida e publicada (2010), e a trazendo para os tempos atuais (2018). Foi verificado que, atualmente, há diversas inovações no campo da utilização de dispositivos reconfiguráveis, com o objetivo de otimizar o deslocamento de usuários e reduzir o tempo de espera, que foram desenvolvidas.

Contudo, analisando-se a quantidade de empresas depositantes e o número de patentes concedidas mundialmente após a data de publicação da patente do CDT, é possível concluir que, se a patente PI 0803135-5 fosse depositada atualmente, não atenderia aos requisitos de novidade e atividade inventiva, e, portanto, não seria concedida. Além disso, considerando-se o nível de maturidade da patente TLR4, ou seja, em nível de protótipo, quando diversas patentes relacionadas já estão sendo fabricadas e comercializadas, torna-se ainda mais questionável a manutenção dessa patente.

Todas as pesquisas e análises realizadas foram consolidadas em uma matriz FOFA, que buscou contextualizar e destacar as principais características da invenção. A matriz elaborada demonstrou que, apesar de ter sido depositada há anos, a invenção está equilibrada e possui uma leve preponderância dos pontos fortes, devido principalmente ao seu grau de maturidade tecnológica.

Após o estudo de prospecção tecnológica, é possível ressaltar a importância dos investimentos em testes operacionais para a invenção, com o objetivo de comparar a invenção com as tecnologias concorrentes presentes no mercado. A partir deste teste, será possível demonstrar a aplicabilidade desta invenção para a otimização de sistemas de elevadores, bem como estimular a busca por estratégias de transferência de tecnologia.

\section{Referências}

AQUARIUS ENGINES. Disponível em: < http://www.aquariusengines.com/> Acesso em: 15 jun. 2018.

BRASIL. Emenda Constitucional n. 95, de 2016. Altera o Ato das Disposições Constitucionais Transitórias, para instituir o Novo Regime Fiscal, e dá outras providências. Brasília, DF: Casa Civil, 16 dez. 2016. Disponível em: <http://www.planalto.gov.br/ccivil_03/constituicao/emendas/emc/ emc95.htm>. Acesso em: 19 jun. 2018.

Lei Complementar $n^{\circ} 131$, de 27 de maio de 2009. Altera a redação da Lei de Responsabilidade Fiscal no que se refere à transparência da gestão fiscal. Brasília, DF: Casa Civil, 27 mai. 2009. Disponível em: http://www.portaltransparencia.gov.br/. Acesso em: 19 jun. 2018.

CABRAL, M. Brasileiro “pai” dos elevadores sem cabo: sistema revolucionário, que transporta as cabines tanto na horizontal quanto na vertical, deve aumentar capacidade de transporte em até 50\%. 2016. Disponível em: <https://epocanegocios.globo.com/Empreendedorismo/noticia/2016/03/ brasileiro-e-pai-dos-elevadores-sem-cabos.html >. Acesso em: 23 abr. 2018.

FERrEirA, A. A.; GUimarÃES, E. R.; CONTADOR, J. C. Patente como instrumento competitivo e como fonte de informação tecnológica. Disponível em: <http://www.scielo.br/pdf/ gp/v16n2/v16n2a05.pdf>. Acesso em: 30 maio 2018.

GALEMBECK, F.; ALMEIDA, W.Propriedade intelectual. Parcerias Estratégicas, n. 20, 2005. 
HITACHI. 2017. Disponível em: <http://social-innovation.hitachi/br/case_studies/elevator_china/ index.html> Acesso em: 13 jun. 2018.

INSTITUTO NACIONAL DE PROPRIEDADE INDUSTRIAL - INPI. Manual para o depositante de patentes. Brasília: INPI.

LOBATO, A. A. A geração de patentes na Universidade Federal de Minas Gerais. 2000.

Dissertação (Mestrado). Ciências da Informação, Belo Horizonte, 2000.

MARTINS, Walter Hélio de Lima. Produção científica - publicação versus patente: o caso CPGEI-UTFPR. Ponta Grossa: [s.n.], 2010.

MITSUBISHI. Disponível em: <http://www.mitsubishielectric.com/news/2017/1101.html> Acesso em: 13 jun. 2018.

\section{NATIONAL AERONAUTICS AND SPACE ADMINISTRATION, NASA: Technology Readiness}

Level - TLR. Agosto de 2017 pelo Oficial da NASA: Brian Dunbar. Disponível em: < https://www. nasa.gov/directorates/heo/scan/engineering/technology/txt_accordion1.html > . Acesso em: 23 maio 2018.

PORTAL SÃO FRANCISCO. Elevador. 2018. Disponível em: <https://www.portalsaofrancisco.com. br/curiosidades/elevador >. Acesso em: 27 abr. 2018.

UNIVERSIDADE DE BRASÍLIA (Brasil). INPI. Carlos Humberto Llanos Quintero. Sistema de Controle Distribuído de Grupo de Elevadores Usando Dispositivos Reconfiguráveis. $\mathrm{BR} \mathrm{n}^{\circ} \mathrm{PI}$ 0803135-5, 02 jul. 2008, 09 mar. 2010. Brasília: UnB, 2010.

WEB OF SCIENCE [Base de dados - Internet]. Clarivate Analytics. 2018. Disponível em: < https:// webofknowledge.com> Acesso em: jun. 2018.

WORLD INTELLECTUAL PROPERTY ORGANIZATION - WIPO - PATENTSCOPE. Disponível em: $<$ https://patentscope.wipo.int/search/pt/search.jsf > . Acesso em: 06 jun. 2018.

\section{Sobre os autores}

\section{Gisele Cristina Borges}

E-mail: giselecb@gmail.com

Mestranda em Propriedade Intelectual e Transferência de Tecnologias para Inovação pela Unb. MBA em Gerenciamento de Projetos pelo IBMEC. Especialista em Análise de Sistemas pela Universidade Católica de Brasília. Endereço Profissional: SIG Quadra 1 Lote 385 - Edifício Platinum Office - 3 Andar - Sala 314 - Brasília - DF, CEP: 70610-480.

\section{Ana Carolina Nerva Blumm}

\section{E-mail: ana.blumm@gmail.com}

Graduada em Engenharia de Produção pela Universidade de Brasília (UnB) em 2015. Mestranda do PROFNIT como aluna especial.

Endereço Profissional: Elogroup Desenvolvimento e Consultoria, ELOGROUP, SGAN 607, Edifício Medical Center, Bloco B, sala 311, Brasília, DF. 


\section{Eduardo Henrique da Silva Figueiredo Matos}

E-mail: eduardo.silva20@gmail.com

Graduado em Administração em Análise de Sistemas.

Endereço Profissional: Ministério da Saúde - Ministério da Saúde - Esplanada dos Ministérios, Bloco G, Térreo;

Brasília/DF - CEP: 70058-900.

\section{Carolyne Caetano Gonçalves}

E-mail: carolynecgoncalves@hotmail.com

Bacharel em Biotecnologia na Universidade de Brasília.

Endereço Profissional: Universidade de Brasília - Edifício CDT - Campus Universitário Darcy Ribeiro, Brasília - DF.

Caixa Postal: 04397. CEP: 70904-970.

\section{Wina Eleana Lages Pereira}

E-mail:wina.pereira@embrapa.br

Graduada em Direito pela Faculdades Integradas do Tapajós ( 2002). Especialista em Direito Público pela Universidade Potiguar (2008). Especialista em Direito Privado pela Universidade Gama Filho (2009).

Endereço Profissional: Parque Estação Biológica - PqEB s/n. - Brasília, DF - Brasil - CEP 70770-901.

\section{Roosevelt Tomé Silva Filho}

E-mail: roosevelt.tome@gmail.com

É Analista de Sistemas e Economista especializado em gerenciamento de projetos, Gestão do Conhecimento, Inteligência Competitiva e Governo Eletrônico. Especialista em Sistemas de Informação em Ciência, Tecnologia e Inclusão Digital.

Endereço Profissional: 3iTC Consultoria - CLN 213 bloco D sala 107 - Brasília DF - CEP: 70872540.

\section{Paulo Gustavo Barboni Dantas Nascimento}

E-mail: pbarboni@unb.br

Bacharel em Química, pela USP (1998). Mestre em Química, pela FFCLRP/USP (2001). Especialista lato sensu em Tecnologias na Aprendizagem, no Centro Universitário Senac (2016). Doutor em Química, na FCFRP, pela Universidade de São Paulo (USP) (2005). Pós-doutor em Farmacologia, pela FMRP (2009).

Endereço Profissional: Universidade de Brasília - Edifício CDT - Campus Ceilândia - QNN14 Área Especial, Ceilândia, DF - CEP 72220-140.

\section{Adriana Regina Martin}

\section{E-mail: adrianamartin@unb.br}

Bacharel e Licenciada em Química pela UFSCar. Mestra e Doutora em Ciência e Engenharia dos Materiais pela UFSCar. Pós-Graduada em Política e Estratégia pela ADESG. Doutorado Sandwich na Universidade de WisconsinMadison/USA com Bolsa Fulbright. Pós-Doutora em Inovação Tecnológica pela UFSCar.

Endereço Profissional: Ministério da Ciência, Tecnologia, Inovações e Comunicações - MCTIC - Esplanada dos Ministérios, Bloco E. CEP: 70067-900 - Brasília - DF. 